\title{
Osimertinib: A Review in Previously Untreated, EGFR Mutation-Positive, Advanced NSCLC
}

\author{
Yvette N. Lamb ${ }^{1}$
}

Published online: 26 September 2021

(c) Springer Nature 2021, corrected publication 2021

\begin{abstract}
Activating mutations in the epidermal growth factor receptor $(E G F R)$ gene have been identified as key oncogenic drivers of non-small cell lung cancer (NSCLC). Osimertinib (Tagrisso ${ }^{\circledR}$ ) is an orally administered, third-generation EGFR tyrosine kinase inhibitor (EGFR-TKI) that is widely approved for the first-line treatment of advanced NSCLC with activating EGFR mutations. In the pivotal phase III FLAURA trial, osimertinib significantly prolonged progression-free survival (PFS) and overall survival (OS) relative to first-generation EGFR-TKIs in patients with previously untreated, EGFR mutation-positive, advanced NSCLC. Osimertinib also significantly prolonged central nervous system (CNS) PFS in patients with CNS metastases at trial entry. Osimertinib had a generally manageable tolerability profile; the majority of adverse events considered to be possibly related to treatment were of mild to moderate severity. Osimertinib represents a valuable targeted therapeutic for use in adults with previously untreated, EGFR mutation-positive, advanced NSCLC.
\end{abstract}

\section{Plain Language Summary}

Non-small cell lung cancer (NSCLC) is the most common type of lung cancer and is associated with a poor prognosis. In about $17 \%$ of Caucasian patients and 39\% of Asian patients with NSCLC, mutations in the epidermal growth factor receptor $(E G F R)$ gene drive tumour growth. EGFR tyrosine kinase inhibitors (EGFR-TKIs) improve clinical outcomes in patients with NSCLC harbouring EGFR mutations. Osimertinib (Tagrisso ${ }^{\circledR}$ ) is the first approved third-generation EGFR-TKI. When given to patients with previously untreated, EGFR mutation-positive, advanced NSCLC, osimertinib delayed disease progression or death by $\approx 9$ months and extended overall survival by $\approx 7$ months relative to first-generation EGFR-TKIs. Overall, the tolerability of osimertinib was similar to that of the first-generation EGFR-TKIs. Osimertinib is an effective, valuable treatment for patients with previously untreated, EGFR mutation-positive, advanced NSCLC.

\section{Digital Features for this Adis Drug Evaluation can be found at} https://doi.org/10.6084/m9.figshare.14788161

The manuscript was reviewed by: $\boldsymbol{K}$. Araki, Department of Medical Oncology, Gunma Prefectural Cancer Center, Ohta, Gunma, Japan; C.-C. Lin, National Taiwan University Hospital, Taipei, Taiwan.

\section{Osimertinib: clinical considerations in untreated,} EGFR mutation-positive, advanced NSCLC

Selectively inhibits mutant EGFR kinase activity

Prolongs PFS and OS relative to first-generation EGFRTKIs

Readily penetrates blood-brain barrier and is active against CNS metastases

Most common potentially drug-related adverse events dermatological or gastrointestinal in nature

Yvette N. Lamb

demail@ springer.com

1 Springer Nature, Private Bag 65901, Mairangi Bay, Auckland 0754, New Zealand 


\section{Introduction}

Non-small cell lung cancer (NSCLC) accounts for the majority $(\approx 85 \%)$ of all lung cancers and is a leading contributor to cancer-related mortality; advanced NSCLC is associated with a 5-year survival rate of $<5 \%[1,2]$. The best-characterized oncogenic drivers of NSCLC include activating mutations in the tyrosine kinase domain of the epidermal growth factor receptor $(E G F R)$ gene, the most common of which are exon 19 deletions and the exon 21 L858R substitution [3]. EGFR mutation prevalence varies based on ethnicity, with mutations being detected in $\approx 17 \%$ of Caucasian patients and $\approx 39 \%$ of Asian patients with NSCLC [2]. These mutations serve as both biomarkers and rational molecular targets for tailored therapy, sensitizing the tumours that harbour them to the effects of EGFR tyrosine kinase inhibitors (EGFR-TKIs) [4].

The emergence of EGFR-TKIs at the beginning of the 21 st century constituted a paradigm shift in the management of EGFR mutation-positive NSCLC, heralding an era of precision medicine. Compared with traditional cytotoxic therapies, EGFR-TKIs have improved clinical outcomes and health-related quality of life in patients with advanced disease [5]. However, acquired resistance to first-generation (e.g. erlotinib, gefitinib) and second-generation (e.g. afatinib, dacomitinib) EGFR-TKIs typically develops after a median of 9-15 months, with the EGFR T790M gatekeeper mutation appearing to mediate the development of resistance in $\approx 50-60 \%$ of patients [6]. Furthermore, the aforementioned EGFR-TKIs (particularly second-generation) non-selectively inhibit wildtype EGFR and can be associated with dose-limiting toxicities [7, 8]. Third-generation EGFR-TKIs have been designed to selectively inhibit the sensitizing and T790M mutant forms of EGFR tyrosine kinase whilst largely sparing wild-type EGFR activity and thus reducing off-target toxicity relative to earliergeneration EGFR-TKIs [7, 8].

Osimertinib (Tagrisso ${ }^{\circledR}$ ) is an orally administered, thirdgeneration EGFR-TKI that is widely approved for the first-line treatment of advanced NSCLC with activating EGFR mutations (Sect. 4). This article reviews the clinical efficacy and tolerability of osimertinib in this indication. An overview of the pharmacological properties of osimertinib is provided in Table 1. While osimertinib has also been approved in other NSCLC treatment settings (e.g. in EGFR-TKI-experienced patients with advanced EGFR T790M mutation-positive NSCLC; reviewed previously [9]) in many countries, use in these indications is beyond the scope of this review.

\section{Therapeutic Efficacy of Osimertinib}

The therapeutic efficacy of osimertinib in the first-line treatment of EGFR mutation-positive, advanced NSCLC was evaluated in the randomized, double-blind, activecontrolled, multinational phase III FLAURA trial (Fig. 1) [10]. Promising results in this treatment setting were initially seen in the phase I component of the AURA trial [11]; AURA will not be discussed further, given that phase III data are available.

FLAURA enrolled adults with locally advanced or metastatic NSCLC who had not previously been treated for advanced disease and were eligible for first-line treatment with a first-generation EGFR-TKI [10]. The presence of the EGFR exon 19 deletion or L858R mutation (alone or in conjunction with other $E G F R$ mutations), confirmed locally or centrally, was also required. Patients with central nervous system (CNS) metastases were eligible if their condition was neurologically stable [10].

Patients were assigned oral osimertinib $(n=279)$ or an oral comparator EGFR-TKI (erlotinib or gefitinib; $n=277$ ) [dosages provided in Table 2], with randomization stratified by tumour EGFR mutation (exon 19 deletion or L858R) and race (Asian or non-Asian) [10]. Treatment continued until disease progression (if deemed to be of clinical benefit, treatment was permitted to continue beyond the point of disease progression), unacceptable toxicity or withdrawal of consent. The primary endpoint was progression-free survival (PFS), determined by investigator assessments based on RECIST version 1.1 criteria. Efficacy analyses were conducted in the full analysis set (FAS), which included all randomized patients [10]. After the primary endpoint analysis, progression event data were no longer centrally collected [12]. A final analysis of overall survival (OS), a secondary endpoint, was planned for after $\approx 318$ deaths in the FAS [12].

Baseline characteristics were generally well balanced between treatment arms [10]. The majority of patients had metastatic disease (95\%), with $35 \%$ having visceral metastases and $21 \%$ having CNS metastases. In each treatment group, $63 \%$ of patients had the EGFR exon 19 deletion at randomization and $37 \%$ had the L858R mutation [10].

\subsection{Progression-Free Survival}

Osimertinib significantly prolonged investigator-assessed PFS relative to comparator EGFR-TKIs (primary endpoint; Table 2) [10]. At data cut-off for the primary analysis, the median duration of follow-up for PFS was 15.0 months in the osimertinib group and 9.7 months in the comparator EGFR-TKI group; RECIST-defined progression or death 


\section{Table 1 Pharmacological properties of osimertinib in advanced NSCLC with activating EGFR mutations}

\section{Pharmacodynamic properties}

Binds irreversibly to clinically relevant mutant EGFR kinase forms (e.g. L858R, ex19del, T790M) to selectively inhibit mutant EGFR kinase activity [7, 19, 20]; potently inhibits EGFR phosphorylation in EGFR-TKI-sensitizing mutant and T790M mutant NSCLC cell lines, exhibiting less activity against wild-type cell lines [7, 20]

Produces two pharmacologically active metabolites; AZ7550 is similar to osimertinib in potency and selectivity, while AZ5104 is more potent in inhibiting both mutant EGFR and wild-type EGFR (smaller selectivity margin than osimertinib) [7, 19]

Dose-dependently induced profound and sustained tumour regression in xenograft and transgenic models of $E G F R$-mutant lung cancer (including those of CNS metastases) in vivo [7, 30]; exhibited potent anti-tumour activity against both L858R and L858R/T790M EGFR-mutant tumours (unlike afatinib, which was only effective against the former) in transgenic mice [7]

Exhibited anti-tumour effects in pts with NSCLC harbouring various EGFR mutations (common and uncommon) in clinical trials [10, 11, 27]; inhibited growth of CNS metastases in pts with EGFR mutation-positive NSCLC [13, 31]

Resistance to osimertinib eventually develops in pts, with various mechanisms underlying acquired resistance [32]; in a subset of pts in the phase III FLAURA trial (Sect. 2), the most common acquired resistance mechanisms identified in osimertinib recipients who experienced clinical progression and/or discontinued treatment were MET amplification $(15 \% ; 14 / 91)$ and the EGFR C797S mutation $(7 \%$; 6/91) [33]

Potential for QTc prolongation and changes in cardiac contractility [18-20]; a concentration-dependent QTc interval prolongation of 14 ms is predicted at the recommended dosage $[19,20]$; in a pooled analysis of data from clinical trials $(n=1479$ treated with osimertinib), a QTc increase from baseline of $>60 \mathrm{~ms}$ and a QTc $>500 \mathrm{~ms}$ occurred in $3.1 \%$ and $0.8 \%$ of pts, respectively, while an LVEF decline of $\geq 10 \%$ from baseline and to $<50 \%$ LVEF occurred in $3.2 \%$ of pts who had baseline data and $\geq 1$ follow-up assessment $(n=1233)[19,20]$

\section{Pharmacokinetic properties}

Linear pharmacokinetics with dose-proportional increases in exposure $\left(\mathrm{C}_{\max }\right.$ and AUC; dose range 20-240 $\left.\mathrm{mg}\right)[19,20,34]$

Slow absorption after oral administration (median $\mathrm{T}_{\max } 6 \mathrm{~h}$ ) [19, 20,34]; no clinically meaningful effects of food on osimertinib exposure in pts with EGFR mutation-positive advanced NSCLC or of omeprazole-induced gastric pH elevations on exposure in healthy volunteers [35]

Steady-state exposures reached after 15 days of once-daily administration (drug accumulation $\approx 3$-fold) [19]

Extensively distributed in tissues (mean $\mathrm{V}_{\mathrm{d}} 918 \mathrm{~L}$ at steady state) [19, 20]; high blood-brain barrier penetrance and brain exposure relative to other EGFR-TKIs in preclinical studies (brain to plasma AUC ratios $\approx 2$ with oral dosing in animal models [19]) [30, 36]; achieved rapid, high and uniform brain exposure in a phase I trial in adults with EGFR mutation-positive advanced NSCLC and CNS metastases $(n=4)$ [31]

Main metabolic pathways are oxidation (primarily by CYP3A) and dealkylation, with two pharmacologically active metabolites found in plasma after oral administration (AZ7550 and AZ5104; each had a geometric mean exposure $\approx 10 \%$ that of osimertinib at steady state) [19, 20, 34]

Low to moderate clearance $(14.2 \mathrm{~L} / \mathrm{h}$ at steady state) and long half-life $(\approx 50 \mathrm{~h}$ after single dose $)$ in pts with advanced NSCLC [34]

Eliminated primarily in faeces $(68 \%)$ and less so in urine (14\%); unchanged drug represented $\approx 2 \%$ of the elimination [19, 20]

Pharmacokinetics in pts with $\mathrm{CL}_{\mathrm{cr}}<15 \mathrm{~mL} / \mathrm{min}$ or severe hepatic impairment (TBil 3-10 $\times$ ULN and any AST) unknown [19, 20]; in the EU, caution is required in pts with $\mathrm{CL}_{\mathrm{cr}}<30 \mathrm{~mL} / \mathrm{min}$ and use in pts with severe hepatic impairment is not recommended [20]

\section{Drug interactions}

Concomitant use of strong CYP3A inducers may decrease osimertinib exposure and should be avoided in the EU [20] and, if possible, in the USA (dosage adjustments required if concomitant use is unavoidable) [19]; in the EU, the co-administration of osimertinib and moderate CYP3A4 inducers should be approached with caution or, if possible, avoided [20]

In the EU, pts concomitantly using drugs with disposition dependent upon BCRP or P-gp and narrow therapeutic indices should be closely monitored for tolerability of the concomitant drug (exposure potentially increased) [20]; in the USA, pts concomitantly using BCRP or P-gp substrates should be monitored for adverse reactions of the BCRP or P-gp substrate (unless instructed otherwise in approved labelling) [19]

Effect of use with drugs known to prolong QTc interval is unknown; in the USA, co-administration of osimertinib and drugs known to prolong the QTc interval with known risk of Torsades de pointes should be avoided if feasible (or periodic ECG monitoring conducted if not) [19]

$A S T$ aspartate aminotransferase, $A U C$ area under the plasma concentration-time curve, $B C R P$ breast cancer resistance protein, $C L_{c r}$ creatinine clearance, $C_{\max }$ peak plasma concentration, $C N S$ central nervous system, EGFR epidermal growth factor receptor, ex19del exon 19 deletion, $L V E F$ left ventricular ejection fraction, NSCLC non-small cell lung cancer, $P$-gp P-glycoprotein, pts patients, TBil total bilirubin, TKI tyrosine kinase inhibitor, $T_{\max }$ time to peak plasma concentration, $U L N$ upper limit of normal, $V_{d}$ volume of distribution

had occurred in $49 \%$ of osimertinib recipients versus $74 \%$ of comparator EGFR-TKI recipients. In a sensitivity analysis, results for PFS according to blinded independent central review (BICR) were consistent with those for PFS based on investigator assessments [10].

The benefit of osimertinib over comparator EGFR-TKIs for PFS was consistent across all predefined subgroups assessed, including those based on EGFR mutation at randomization (exon 19 deletion vs L858R), known or treated
CNS metastases at trial entry (yes vs no) and race (Asian vs non-Asian) [all hazard ratios (HRs) and upper limits of associated $95 \%$ CIs < 1] [10]. In patients with known or treated CNS metastases, median PFS was 15.2 months in the osimertinib group versus 9.6 months in the comparator EGFR-TKI group (HR 0.47; 95\% CI 0.30-0.74; $p<0.001$ ); in patients without CNS metastases, median PFS was 19.1 months versus 10.9 months in the corresponding groups (HR 0.46 ; 95\% CI $0.36-0.59 ; p<0.001)$ [10]. 


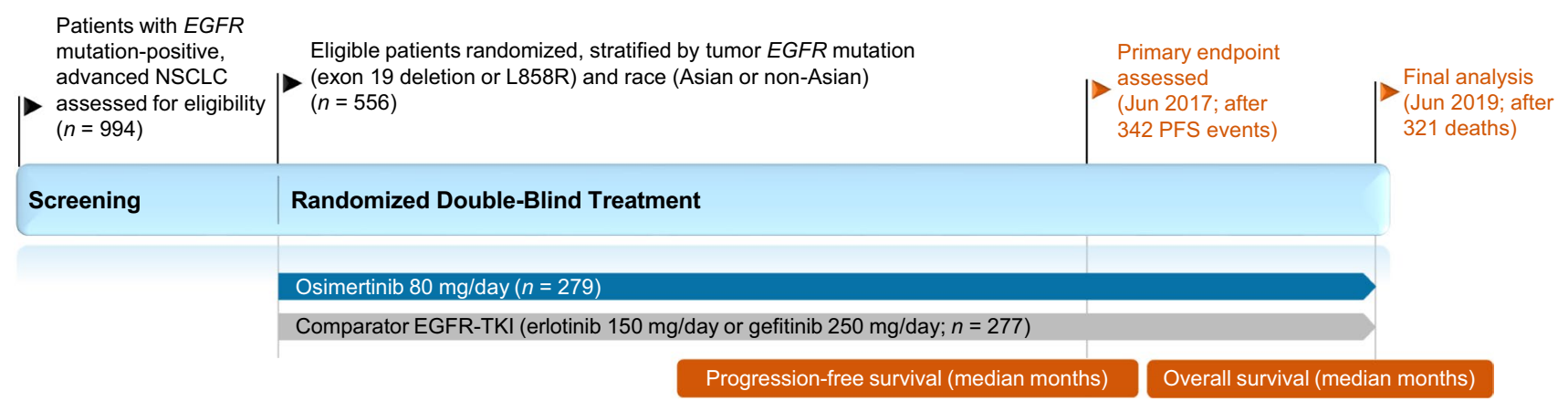

Fig. 1. Trial design of the randomized, double-blind, multinational phase III FLAURA trial in adults with EGFR mutation-positive, advanced NSCLC [10, 12]. Efficacy results are reported in the ani-

Table 2 Efficacy of osimertinib in the first-line treatment EGFR mutation-positive advanced NSCLC in FLAURA

Osimertinib $^{\mathrm{a}}$

Comparator EGFR-TKIs ${ }^{\mathrm{b}}$

\begin{tabular}{|c|c|c|}
\hline \multicolumn{3}{|l|}{ Full analysis set $[10,12]$} \\
\hline No. of pts & 279 & 277 \\
\hline Median PFS ${ }^{\mathrm{c}}$ (months) & 18.9 & 10.2 \\
\hline Hazard ratio $(95 \% \mathrm{CI})$ & \multicolumn{2}{|l|}{$0.46(0.37-0.57)^{* * *}$} \\
\hline Median OS ${ }^{\mathrm{d}}$ (months) & 38.6 & 31.8 \\
\hline Hazard ratio $(95.05 \% \mathrm{CI})$ & \multicolumn{2}{|l|}{$0.80(0.64-1.00)^{*}$} \\
\hline ORR (\% of pts) & 80 & 76 \\
\hline Odds ratio $(95 \% \mathrm{CI})$ & \multicolumn{2}{|l|}{$1.27(0.85-1.90)$} \\
\hline \multicolumn{3}{|l|}{ CNS full analysis set ${ }^{e}$ [13] } \\
\hline No. of pts & 61 & 67 \\
\hline Median CNS PFS ${ }^{f}$ (months) & NR & 13.9 \\
\hline Hazard ratio $(95 \% \mathrm{CI})$ & \multicolumn{2}{|l|}{$0.48(0.26-0.86)^{* *}$} \\
\hline CNS ORR (\% of pts) & 66 & 43 \\
\hline Odds ratio $(95 \% \mathrm{CI})$ & \multicolumn{2}{|l|}{$2.5(1.2-5.2)^{* *}$} \\
\hline
\end{tabular}

Data cut-off 12 June 2017 for all endpoints except OS (data cut-off 25 June 2019). PFS and ORR based on investigator assessment; CNS PFS and ORR based on neuroradiological BICR

$B I C R$ blinded independent central review, $C N S$ central nervous system, $E G F R$ epidermal growth factor receptor, $N R$ not reached, $O R R$ objective response rate, $O S$ overall survival, $P F S$ progression-free survival, pts patients, TKI tyrosine kinase inhibitor

${ }^{*} p=0.046,{ }^{* *} p \leq 0.014,{ }^{* * *} p<0.001$ vs comparator EGFR-TKIs

${ }^{a} 80 \mathrm{mg}$ once daily

${ }^{\mathrm{b}}$ Gefitinib $250 \mathrm{mg}$ ( $n=183$ in full analysis set) or erlotinib $150 \mathrm{mg}(n$ $=94$ ) once daily

${ }^{\mathrm{c}} \mathrm{PFS}$ was primary endpoint; time from randomization to objective disease progression or death (any cause)

${ }^{\mathrm{d}}$ At the time of final OS analysis (alpha value 0.0495 to maintain overall 5\% type 1 error rate across interim and final OS analyses)

${ }^{\mathrm{e}}$ All pts with baseline CNS metastases (measurable or not) by BICR

${ }^{\mathrm{f}}$ Time from randomization to objective CNS progression or death (any cause) mated figure (available online). EGFR epidermal growth factor receptor, $H R$ hazard ratio, NSCLC non-small cell lung cancer, $P F S$ progression-free survival, TKI tyrosine kinase inhibitor

\subsection{Overall Survival}

At the time of the primary analysis of PFS, median OS could not be calculated in either group (HR for death 0.63 ; 95\% CI 0.45-0.88; $p=0.007)$; in this interim OS analysis, a $p$-value $<0.0015$ was required for statistical significance [10]. At 12 months, $89 \%$ of osimertinib recipients were alive versus $82 \%$ of comparator EGFR-TKI recipients; at 18 months, $83 \%$ versus $71 \%$ were alive [10].

Osimertinib recipients had significantly longer OS than comparator EGFR-TKI recipients at the time of the final OS analysis (Table 2) [12]. At data cut-off, the median duration of follow-up for OS was 35.8 months in the osimertinib group and 27.0 months in the comparator EGFRTKI group. At 24 months, $74 \%$ of osimertinib recipients were alive versus $59 \%$ of comparator EGFR-TKI recipients; at 36 months, $54 \%$ versus $44 \%$ were alive. The OS benefit with osimertinib versus comparator EGFR-TKIs was generally consistent across predefined subgroups; all HRs were $<1$, except for analyses in Asian patients (HR 1.00; 95\% CI 0.75-1.32) and patients with the L858R mutation at randomization (HR 1.00; 95\% CI 0.71-1.40) [12].

\subsection{Other Outcomes}

Objective response rate (ORR) did not markedly differ between osimertinib and comparator EGFR-TKI recipients (Table 2) [10]. While the median time to response was 6.1 weeks in each treatment group, the median duration of response was longer with osimertinib than with comparator EGFR-TKIs [17.2 months (95\% CI 13.8-22.0) vs 8.5 months (95\% CI 7.3-9.8)]. The median best percentage change in target-lesion size was $-54.7 \%$ with osimertinib versus $-48.5 \%$ with comparator EGFR-TKIs $(p=0.003)$ and disease control was achieved in $97 \%$ of osimertinib 
recipients versus $92 \%$ of comparator EGFR-TKI recipients [odds ratio (OR) 2.78; 95\% CI 1.25-6.78; $p=0.01$ ] [10].

Osimertinib was active against CNS metastases, significantly prolonging CNS PFS relative to comparator EGFRTKIs in patients with CNS metastases at trial entry (Table 2) [13]. At data cut-off, the median duration of follow-up for CNS PFS was 12.4 months in the osimertinib group and 7.0 months in the comparator EGFR-TKI group. CNS progression by BICR occurred in $20 \%$ of osimertinib recipients versus $39 \%$ of comparator EGFR-TKI recipients and the CNS ORR was higher with osimertinib than with comparator EGFR-TKIs (Table 2) [13]. In the FAS, which included patients with and without CNS metastases at trial entry, investigator-assessed CNS progression events were observed in $6 \%$ of osimertinib recipients versus $15 \%$ of comparator EGFR-TKI recipients [10].

First-line osimertinib may delay the need for subsequent therapies relative to comparator EGFR-TKI therapy, with $28 \%$ of the osimertinib group versus $9 \%$ of the comparator EGFR-TKI group continuing to receive the first-line trial drug at 36 months [12]. At the time of the final analysis of OS, $48 \%$ of osimertinib recipients and $65 \%$ of comparator EGFR-TKI recipients had started a first subsequent anticancer therapy after discontinuing their randomized treatment (with $47 \%$ of the latter group having received osimertinib as their first subsequent therapy). The median time to first subsequent therapy or death was 25.5 months with osimertinib versus 13.7 months with comparator EGFR-TKIs (HR 0.48; 95\% CI 0.39-0.58; $p<0.0001)$. Of all randomized patients, $26 \%$ of osimertinib recipients and $33 \%$ of comparator EGFRTKI recipients received a second subsequent therapy. The median time to second subsequent therapy was 31.1 months with osimertinib versus 23.4 months with comparator EGFR-TKIs (HR 0.69; 95\% CI 0.56-0.84; $p=0.0003$ ) [12].

With respect to patient-reported outcomes, both osimertinib and comparator EGFR-TKI recipients reported improvements in key lung cancer symptoms (i.e. cough, chest pain, dyspnoea, fatigue and appetite loss), global health status/ quality of life and levels of functioning from baseline to treatment discontinuation [14]. There were no statistically significant, clinically relevant differences in mean changes between treatment groups [14].

\subsection{Efficacy in Asian Patients}

In subsets of Asian patients $(n=162$ and 160 randomized to osimertinib and comparator EGFR-TKI therapy at Asian sites) [15] and Japanese patients ( $n=65$ and 55 randomized to osimertinib and gefitinib) [16] in FLAURA, the efficacy of osimertinib relative to comparator EGFR-TKI therapy was generally comparable to that in the overall study population. In the Asian subset, median investigator-assessed PFS was 16.5 months in osimertinib recipients versus 11.0 months in comparator EGFR-TKI recipients (HR 0.54; 95\% CI $0.41-0.72 ; p<0.0001$ ) [15]. The ORR was $80 \%$ with osimertinib and $75 \%$ with comparator EGFR-TKIs. Deaths occurred in $20 \%$ and $28 \%$ of osimertinib and comparator EGFR-TKI recipients, with OS data immature at the time of this analysis; median OS was not reached in either treatment arm. At 18 months, the survival rate was $82 \%$ with osimertinib versus $72 \%$ with comparator EGFR-TKIs (HR for death 0.65 ; 95\% CI 0.42-1.02) [15]. In the Japanese subset, median investigator-assessed PFS was 19.1 months with osimertinib versus 13.8 months with gefitinib (HR $0.61 ; 95 \%$ CI 0.38-0.99; nominal $p=0.0456$ ) [16]. The ORR was $75 \%$ with osimertinib versus $76 \%$ with gefitinib. Deaths occurred in $14 \%$ and $18 \%$ of osimertinib and gefitinib recipients, with OS data immature at the time of analysis [16].

FLAURA China evaluated first-line osimertinib in Chinese patients with EGFR mutation-positive advanced NSCLC enrolled in either the global FLAURA study or in a China-only study following the same protocol as FLAURA (total $n=71$ and 65 randomized to osimertinib and gefitinib) [17]. In FLAURA China, median investigator-assessed PFS was 17.8 months in osimertinib recipients versus 9.8 months in gefitinib recipients (HR 0.56; 95\% CI 0.37-0.85; nominal $p=0.007$ ) [primary endpoint]; median follow-up for PFS was 16.7 months and 9.8 months in the respective groups. At data cut-off for the final OS analysis, median OS was 33.1 months with osimertinib versus 25.7 months with gefitinib (HR 0.85; 95\% CI 0.56-1.29). Deaths had occurred in 63\% and $68 \%$ of osimertinib and gefitinib recipients. The median follow-up for OS was 31.0 months and 24.9 months in the respective groups [17].

\section{Tolerability of Osimertinib}

Osimertinib had a generally manageable tolerability profile in adults with previously untreated, EGFR mutation-positive advanced NSCLC participating in the pivotal FLAURA trial [10]. The safety of osimertinib in Asian patient subsets (including in the FLAURA China study) was generally consistent with that in the overall FLAURA population [15-17].

At data cut-off for the primary PFS analysis of FLAURA, the median duration of treatment exposure was 16.2 months for osimertinib recipients and 11.5 months for comparator EGFR-TKI recipients; $51 \%$ of osimertinib recipients versus $23 \%$ of comparator EGFR-TKI recipients continued to receive their assigned trial treatment [10]. In osimertinib recipients, the most common adverse events (AEs) assessed by the investigator as possibly related to treatment were rashes and acnes, diarrhoea, dry skin, paronychia, stomatitis, pruritus, decreased appetite, aspartate aminotransferase (AST) elevation and alanine aminotransferase (ALT) elevation (Fig. 2). Grade $\geq 3$ AEs of any causality occurred in 


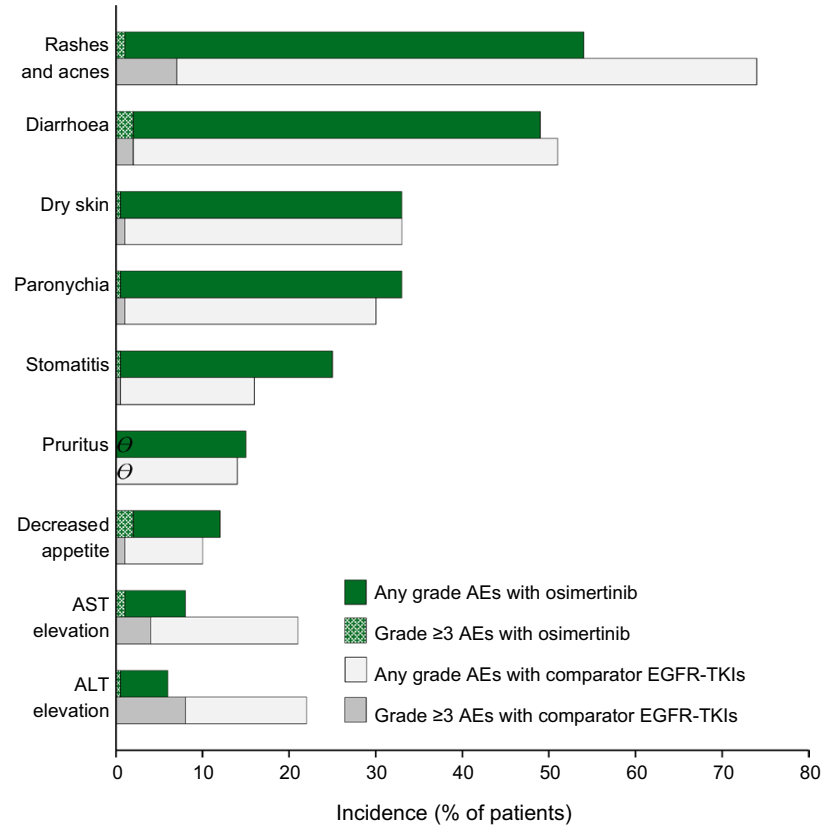

Fig. 2. Adverse events possibly causally related to treatment and occurring in $\geq 10 \%$ of patients in either treatment arm in the FLAURA trial ( $n=279$ and 277 receiving osimertinib and comparator EGFR-TKIs) [10]. AEs adverse events, $A L T$ alanine aminotransferase, AST aspartate aminotransferase, EGFR-TKIs epidermal growth factor receptor tyrosine kinase inhibitors, $\theta$ zero incidence of grade $\geq 3$ AEs

$34 \%$ of osimertinib recipients versus $45 \%$ of comparator EGFR-TKI recipients, with the most common grade $3 \mathrm{AE}$ in the osimertinib group being decreased appetite (3 vs $2 \%$ with comparator EGFR-TKIs). Grade 4 AEs occurred in $2 \%$ of osimertinib recipients and $4 \%$ of comparator EGFR-TKI recipients (no grade 4 AEs in osimertinib recipients were assessed to be possibly related to treatment, while $1 \%$ of comparator EGFR-TKI recipients experienced possibly treatment-related grade 4 ALT elevations). Serious AEs occurred in $22 \%$ of osimertinib recipients versus $25 \%$ of comparator EGFR-TKI recipients and were of interstitial lung disease (ILD) in 2.2 versus $1.4 \%$. AEs led to permanent treatment discontinuation in $13 \%$ of osimertinib recipients versus $18 \%$ of comparator EGFR-TKI recipients, dose interruption in $25 \%$ versus $24 \%$, and dose reduction in $4 \%$ versus $5 \%$. Fatal AEs occurred in $2 \%$ of osimertinib recipients and $4 \%$ of comparator EGFR-TKI recipients [10].

No new safety signals were observed at the time of the FLAURA final OS analysis [12]. Despite a longer median duration of treatment exposure in the osimertinib group than the comparator EGFR-TKI group (20.7 vs 11.5 months), rates of grade $\geq 3$ AEs (42 vs $47 \%$ of patients), serious AEs (27 vs 27\%) and treatment discontinuation due to AEs (15 vs $18 \%$ ) were comparable between treatment groups. Fatal AEs occurred in $3 \%$ of osimertinib recipients versus $4 \%$ of comparator EGFR-TKI recipients and no deaths in the osimertinib group were considered to be related to treatment (vs two in the comparator EGFR-TKI group) [12].

A comprehensive evaluation of cardiac safety data in patients with advanced NSCLC from the osimertinib global safety database did not identify any causal relationship between osimertinib and cardiac failure [18]. Across the clinical study program, most cardiac failure-related AEs were asymptomatic decreases in ejection fraction (Table 1). Generally, ejection fraction decreases resolved without treatment or dose modification of osimertinib. In FLAURA, there was one case of cardiac failure possibly related to osimertinib (occurring in a patient with a medical history of hypertension and chronic heart failure) and one case of pulmonary edema considered to be unrelated to osimertinib [18].

Various warnings pertain to the use of osimertinib and precautions must be taken to reduce the risk or severity of potential AEs [19, 20]. In the USA, cardiac monitoring should be conducted in patients with cardiac risk factors or at increased risk of QTc prolongation (e.g. due to unavoidable concomitant administration with drugs known to prolong QTc interval; Table 1) [19]. This monitoring should also be considered in the EU (and administration in patients with congenital long QT syndrome avoided where possible) [20]. Cardiac adverse reactions may necessitate withholding, dose modification or discontinuation of osimertinib (see local prescribing information) $[19,20]$. In a pooled analysis of data from the FLAURA, AURA and ADAURA trials $(n=1479$ treated with osimertinib), ILD or ILD-like adverse reactions (e.g. pneumonitis) occurred in 3.7\% of osimertinib recipients and were fatal in $0.3 \%[19,20]$. Patients with worsening (or new [20]) symptoms potentially indicative of ILD (e.g. dyspnoea, fever, cough) should be carefully assessed for ILD [19, 20]. Treatment with osimertinib should be withheld during this assessment and discontinued if ILD is confirmed. Based on preclinical data and its mechanism of action, osimertinib can cause foetal harm if used during pregnancy $[19,20]$.

\section{Dosage and Administration of Osimertinib}

Osimertinib is approved for use in the first-line treatment of patients with EGFR mutation-positive, advanced NSCLC in several countries, including those of the EU [20] and the USA [19]. In the EU, osimertinib is indicated as monotherapy for the first-line treatment of adults with locally advanced or metastatic NSCLC with activating EGFR mutations [20]. In the USA, osimertinib is indicated for the firstline treatment of patients with metastatic NSCLC whose 
tumours harbour EGFR mutations (exon 19 deletions or exon 21 L858R mutations) [19]. EGFR mutation status in plasma or tumour specimens should be confirmed using a validated [20] or locally approved [19] test method. Osimertinib is available as 80 or $40 \mathrm{mg}$ tablets, to be swallowed whole with water $[19,20]$. The recommended dose of osimertinib is $80 \mathrm{mg}$ once daily, with treatment continuing until disease progression or unacceptable toxicity. If individual safety and tolerability necessitates a dose reduction, the dose should be reduced to $40 \mathrm{mg}$ once daily $[19,20]$. Co-administering osimertinib with St. John's Wort is contraindicated in the EU [20]. Consult local prescribing information for detailed information concerning the administration of osimertinib in patients who have difficulty swallowing solids, missed doses, management of adverse reactions, contraindications, warnings and precautions, and use in special populations.

\section{Place of Osimertinib in the Management of Previously Untreated, EGFR Mutation-Positive NSCLC}

EGFR-TKIs have revolutionized the treatment of EGFR mutation-positive NSCLC. Osimertinib is the first thirdgeneration EGFR-TKI to be approved for use in the firstline treatment of patients with EGFR mutation-positive, advanced NSCLC. In the USA, osimertinib is recommended as the preferred first-line treatment option (ahead of earliergeneration EGFR-TKIs) for patients with sensitizing EGFR mutation-positive advanced NSCLC (NCCN clinical practice guidelines [21]) and patients with stage IV NSCLC harbouring the EGFR L858R or exon 19 deletion mutation with or without T790M [ASCO/OH(CCO) guidelines [22]]. The European Society for Medical Oncology also considers osimertinib to be the preferred first-line option for patients with advanced/metastatic NSCLC with sensitizing EGFR mutations [3]. Robust testing methods are available for the detection of EGFR mutations in plasma and tissue samples [23].

Osimertinib was significantly more effective than firstgeneration EGFR-TKIs in prolonging PFS and OS in the pivotal FLAURA trial in patients with previously untreated, EGFR mutation-positive advanced NSCLC (Sect. 2). Improvements in PFS and OS were clinically meaningful, with gains of $\approx 9$ months and $\approx 7$ months, respectively. EGFR mutations are more prevalent in Asian populations (Sect. 1) and the efficacy of osimertinib relative to comparator EGFR-TKIs in Asian patient subsets of FLAURA and FLAURA China (Sect. 2.4) was generally comparable to that in the overall FLAURA population. Consistent with preclinical data demonstrating relatively high blood-brain barrier penetration and CNS exposure with osimertinib versus other EGFR-TKIs (Table 1), osimertinib significantly prolonged CNS PFS relative to comparator EGFR-TKI therapy (Sect. 2.3). Activity against CNS metastases is of particular importance given the high rate of CNS metastases in patients with EGFR mutation-positive advanced NSCLC $(\approx 25 \%$ at the time of diagnosis [24]). CNS-penetrant targeted therapies such as osimertinib may delay the need for cranial radiotherapy, which is associated with risks of cognitive impairment $[25,26]$. While the FLAURA trial only enrolled patients with the most common EGFR mutations (exon 19 deletion and L858R), preclinical data (Table 1) and preliminary clinical results [27] indicate that the therapeutic efficacy of osimertinib is likely to extend to tumours harbouring relatively rare EGFR mutations. As with earliergeneration EGFR-TKIs, it should be noted that resistance to osimertinib does eventually develop (Table 1); trials into the mechanisms of acquired resistance are ongoing [e.g. ELIOS (NCT03239340)].

Osimertinib had a generally manageable tolerability profile in patients with previously untreated, EGFR mutationpositive advanced NSCLC (Sect. 3). The safety and tolerability of osimertinib in FLAURA was largely consistent with that established in EGFR-TKI-experienced patients with T790M mutation-positive advanced NSCLC [9]. In FLAURA, the overall tolerability of osimertinib was similar to that of comparator EGFR-TKIs, with a somewhat lower frequency of grade $\geq 3$ AEs (Sect. 3). The majority of AEs possibly causally related to treatment with osimertinib were of mild to moderate severity, the most common being dermatological (e.g. acne and rash, dry skin, paronychia) or gastrointestinal (e.g. diarrhoea, decreased appetite). Patients with a history of ILD or radiation pneumonitis requiring steroid treatment, or any evidence of clinically active ILD, were excluded from clinical trials of osimertinib, as were patients with clinically important cardiac abnormalities [20].

In the absence of direct comparisons between osimertinib and the second-generation EGFR-TKIs, network metaanalyses generally suggest osimertinib has favourable efficacy compared with other first-line EGFR-TKIs (e.g. [28, 29]). Unlike osimertinib, afatinib and dacomitinib may be associated with higher risks of rash and diarrhoea than other EGFR-TKIs [28]. The results of such comparisons must be interpreted with caution, however, given their indirect nature and direct head-to-head comparative studies are desirable.

As an effective first-line treatment for EGFR mutationpositive, advanced NSCLC, with a generally manageable tolerability profile, osimertinib represents a valuable targeted therapeutic for use in adults with previously untreated, EGFR mutation-positive, advanced NSCLC. 


\section{Data Selection Osimertinib: 300 records identified}

Duplicates removed

Excluded during initial screening (e.g. press releases; news reports; not relevant drug/indication; preclinical study; reviews; case reports; not randomized trial)

Excluded during writing (e.g. reviews; duplicate data; small patient number; nonrandomized/phase I/II trials)

\section{Cited efficacv/tolerability articles}

Cited articles not efficacy/tolerability

Search Strategy: EMBASE, MEDLINE and PubMed from 2018 to present. Previous Adis Drug Evaluation published in 2018 was hand-searched for relevant data. Clinical trial registries/ databases and websites were also searched for relevant data. Key words were osimertinib, Tagrisso, metastatic NSCLC. Records were limited to those in English language. Searches last updated 12 August 2021.

Supplementary Information The online version contains supplementary material available at https://doi.org/10.1007/s11523-021-00839-w.

Acknowledgments During the peer review process, the manufacturer of osimertinib was also offered an opportunity to review this article. Changes resulting from comments received were made on the basis of scientific and editorial merit.

\section{Declarations}

Funding The preparation of this review was not supported by any external funding.

Authorship and Conflict of interest Yvette Lamb is a salaried employee of Adis International Ltd/Springer Nature, and declares no relevant conflicts of interest. All authors contributed to the review and are responsible for the article content.

Ethics approval, Consent to participate, Consent to publish, Availability of data and material, Code availability Not applicable.

Open Access This article is licensed under a Creative Commons Attribution-NonCommercial 4.0 International License, which permits any non-commercial use, sharing, adaptation, distribution and reproduction in any medium or format, as long as you give appropriate credit to the original author(s) and the source, provide a link to the Creative Commons licence, and indicate if changes were made. The images or other third party material in this article are included in the article's Creative Commons licence, unless indicated otherwise in a credit line to the material. If material is not included in the article's Creative Commons licence and your intended use is not permitted by statutory regulation or exceeds the permitted use, you will need to obtain permission directly from the copyright holder. To view a copy of this licence, visit http://creativecommons.org/licenses/by-nc/4.0/.

\section{References}

1. Wang S, Cang S, Liu D. Third-generation inhibitors targeting EGFR T790M mutation in advanced non-small cell lung cancer. J Hematol Oncol. 2016;9:34.

2. Zhang Y-L, Yuan J-Q, Wang K-F, et al. The prevalence of EGFR mutation in patients with non-small cell lung cancer: a systematic review and meta-analysis. Oncotarget. 2016;7(48):78985-93.

3. ESMO Guidelines Committee. Metastatic non-small cell lung cancer: ESMO clinical practice guidelines for diagnosis, treatment and follow-up. 2020. https://www.esmo.org/. Accessed 12 Aug 2021.

4. Tan CS, Gilligan D, Pacey S. Treatment approaches for EGFRinhibitor-resistant patients with non-small-cell lung cancer. Lancet Oncol. 2015; 16:e447-59.

5. Greenhalgh J, Boland A, Bates V, et al. First-line treatment of advanced epidermal growth factor receptor (EGFR) mutation positive non-squamous non-small cell lung cancer. Cochrane Database Syst Rev. 2021(3):CD010383.

6. Tan C-S, Kumarakulasinghe NB, Huang Y-Q, et al. Third generation EGFR TKIs: current data and future directions. Mol Cancer. 2018;17:29.

7. Cross DAE, Ashton SE, Ghiorghiu S, et al. AZD9291, an irreversible EGFR TKI, overcomes T790M-mediated resistance to EGFR inhibitors in lung cancer. Cancer Discov. 2014;4(9):1046-61.

8. Shah R, Lester JF. Tyrosine kinase inhibitors for the treatment of $E G F R$ mutation-positive non-small-cell lung cancer: a clash of the generations. Clin Lung Cancer. 2020;21:e216-28.

9. Lamb YN, Scott LJ. Osimertinib: a review in T790M-positive advanced non-small cell lung cancer. Target Oncol. 2017;12(4):555-62.

10. Soria J-C, Ohe Y, Vansteenkiste J, et al. Osimertinib in untreated $E G F R$-mutated advanced non-small-cell lung cancer. N Engl J Med. 2018;378(2):113-25.

11. Ramalingam SS, Yang JC, Lee CK, et al. Osimertinib as first-line treatment of EGFR mutation-positive advanced non-small-cell lung cancer. J Clin Oncol. 2018;36(9):841-9.

12. Ramalingam SS, Vansteenkiste J, Planchard D, et al. Overall survival with osimertinib in untreated, $E G F R$-mutated advanced NSCLC. N Engl J Med. 2020;382(1):41-50.

13. Reungwetwattana $\mathrm{T}$, Nakagawa $\mathrm{K}$, Cho BC, et al. CNS response to osimertinib versus standard epidermal growth factor receptor tyrosine kinase inhibitors in patients with untreated $E G F R$ mutated advanced non-small-cell lung cancer. J Clin Oncol. 2018;36(33):3290-7.

14. Leighl NB, Karaseva N, Nakagawa K, et al. Patient-reported outcomes from FLAURA: osimertinib versus erlotinib or gefitinib in patients with EGFR-mutated advanced non-small-cell lung cancer. Eur J Cancer. 2020;125:49-57.

15. Cho BC, Chewaskulyong B, Lee KH, et al. Osimertinib versus standard of care EGFR TKI as first-line treatment in patients with EGFRm advanced NSCLC: FLAURA Asian subset. J Thorac Oncol. 2019;14(1):99-106.

16. Ohe Y, Imamura F, Nogami N, et al. Osimertinib versus standard-of-care EGFR-TKI as first-line treatment for EGFRm advanced NSCLC: FLAURA Japanese subset. Jpn J Clin Oncol. 2019;49(1):29-36.

17. Cheng $\mathrm{Y}, \mathrm{He} \mathrm{Y}, \mathrm{Li}$ W, et al. Osimertinib versus comparator EGFR TKI as first-line treatment for EGFR-mutated advanced NSCLC: FLAURA China, a randomized study. Target Oncol. 2021;16:165-76.

18. Ewer MS, Tekumalla SH, Walding A, et al. Cardiac safety of osimertinib: a review of data. J Clin Oncol. 2021;39(4):328-37. 
19. AstraZeneca Pharmaceuticals. Tagrisso (osimertinib): US highlights of prescribing information. 2020. http://www.tagrisso.com/. Accessed 12 Aug 2021.

20. AstraZeneca Pharmaceuticals. Tagrisso (osimertinib): EMA summary of product characteristics. 2021. http://www.ema.europa.eu/. Accessed 12 Aug 2021.

21. National Comprehensive Cancer Network ${ }^{\circledR}$. Clinical practice guidelines in oncology ( $\mathrm{NCCN}$ Guidelines $\left.{ }^{\circledR}\right)$ : non-small cell lung cancer (version 4.2021). 2021. http://www.nccn.org/. Accessed 12 Aug 2021.

22. Hanna NH, Robinson AG, Temin S, et al. Therapy for stage IV non-small-cell lung cancer with driver alterations: ASCO and $\mathrm{OH}$ (CCO) joint guideline update. J Clin Oncol. 2021;39(9):1040-91.

23. Gray JE, Okamoto I, Sriuranpong V, et al. Tissue and plasma EGFR mutation analysis in the FLAURA trial: osimertinib versus comparator EGFR tyrosine kinase inhibitor as first-line treatment in patients with EGFR-mutated advanced non-small cell lung cancer. Clin Cancer Res. 2019;25(22):6644-52.

24. Rangachari D, Yamaguchi N, VanderLaan PA, et al. Brain metastases in patients with $E G F R$-mutated or $A L K$-rearranged nonsmall-cell lung cancers. Lung Cancer. 2015;88(1):108-11.

25. Planchard D, Popat S, Kerr K, et al. Metastatic non-small cell lung cancer: ESMO clinical practice guidelines for diagnosis, treatment and follow-up. Ann Oncol. 2018;29(Suppl. 4):iv192-237.

26. Venur VA, Chukwueke UN, Lee EQ. Advances in management of brain and leptomeningeal metastases. Curr Neurol Neurosci Rep. 2020;20(7):26-35.

27. Cho JH, Lim SH, An HJ, et al. Osimertinib for patients with nonsmall-cell lung cancer harboring uncommon EGFR mutations: a multicenter, open-label, phase II trial (KCSG-LU15-09). J Clin Oncol. 2020;38(5):488-95.

28. Holleman MS, Van Tinteren H, Groen HJM, et al. First-line tyrosine kinase inhibitors in EGFR mutation-positive non-small-cell lung cancer: a network meta-analysis. OncoTargets Ther. 2019;12:1413-21.

29. Zhao Y, Liu J, Cai X, et al. Efficacy and safety of first line treatments for patients with advanced epidermal growth factor receptor mutated, non-small cell lung cancer: systematic review and network meta-analysis. BMJ. 2019;367:15460.

30. Ballard P, Yates JWT, Yang Z, et al. Preclinical comparison of osimertinib with other EGFR-TKIs in EGFR-mutant NSCLC brain metastases models, and early evidence of clinical brain metastases activity. Clin Cancer Res. 2016;22(20):5130-40.

31. Ekman S, Cselényi Z, Varrone A, et al. A PET and MRI study exploring osimertinib brain exposure and efficacy in EGFRm NSCLC CNS metastases [abstract no. P76.72]. J Thorac Oncol. 2021;16(3 Suppl.):S620.

32. Ortiz-Cuaran S, Scheffler M, Plenker D, et al. Heterogeneous mechanisms of primary and acquired resistance to third-generation EGFR inhibitors. Clin Cancer Res. 2016;22(19):4837-47.

33. Ramalingam SS, Cheng Y, Zhou C, et al. Mechanisms of acquired resistance to first-line osimertinib: preliminary data from the phase III FLAURA study [abstract no. LBA50]. Ann Oncol. 2018;29(Suppl. 8):viii740.

34. Planchard D, Brown KH, Kim D-W, et al. Osimertinib Western and Asian clinical pharmacokinetics in patients and healthy volunteers: implications for formulation, dose, and dosing frequency in pivotal clinical studies. Cancer Chemother Pharmacol. 2016;77(4):767-76.

35. Vishwanathan K, Dickinson PA, Bui K, et al. The effect of food or omeprazole on the pharmacokinetics of osimertinib in patients with non-small-cell lung cancer and in healthy volunteers. J Clin Pharmacol. 2018;58(4):474-84.

36. Colclough N, Chen K, Johnström P, et al. Preclinical comparison of the blood-brain barrier permeability of osimertinib with other EGFR TKIs. Clin Cancer Res. 2021;27(1):189-201. 